\title{
Endoplasmic Reticulum Stress: Implications for Neuropsychiatric Disorders
}

\author{
Ather Muneer ${ }^{1, *}$ and Rana Mozammil Shamsher Khan ${ }^{2}$ \\ ${ }^{1}$ Islamic International Medical College, Riphah International University, Rawalpindi, ${ }^{2}$ Government Khawaja Safdar Medical College, \\ Sialkot, Pakistan
}

The Endoplasmic reticulum (ER), an indispensable sub-cellular component of the eukaryotic cell carries out essential functions, is critical to the survival of the organism. The chaperone proteins and the folding enzymes which are multi-domain ER effectors carry out 3-dimensional conformation of nascent polypeptides and check misfolded protein aggregation, easing the exit of functional proteins from the ER. Diverse conditions, for instance redox imbalance, alterations in ionic calcium levels, and inflammatory signaling can perturb the functioning of the ER, leading to a build-up of unfolded or misfolded proteins in the lumen. This results in ER stress, and aiming to reinstate protein homeostasis, a well conserved reaction called the unfolded protein response (UPR) is elicited. Equally, in protracted cellular stress or inadequate compensatory reaction, UPR pathway leads to cell loss. Dysfunctional ER mechanisms are responsible for neuronal degeneration in numerous human diseases, for instance Alzheimer's, Parkinson's and Huntington's diseases. In addition, mounting proof indicates that ER stress is incriminated in psychiatric diseases like major depressive disorder, bipolar disorder, and schizophrenia. Accumulating evidence suggests that pharmacological agents regulating the working of ER may have a role in diminishing advancing neuronal dysfunction in neuropsychiatric disorders. Here, new findings are examined which link the foremost mechanisms connecting ER stress and cell homeostasis. Furthermore, a supposed new pathogenic model of major neuropsychiatry disorders is provided, with ER stress proposed as the pivotal step in disease development.

Key Words: Endoplasmic Reticulum; Unfolded Protein Response; Proteostasis Deficiencies; Apoptosis; Biological Psychiatry

This is an Open Access article distributed under the terms of the Creative Commons Attribution Non-Commercial License (http://creativecommons.org/licenses/by-nc/4.0) which permits unrestricted non-commercial use, distribution, and reproduction in any medium, provided the original work is properly cited.

\section{Article History:}

Received September 4, 2018

Revised October 11, 2018

Accepted November 9, 2018

\section{HIGHLIGHTS}

- Unfolded protein response (UPR) is the mechanism whereby eukaryotic cells ensure three dimensional conformation of secreted proteins which is essential for functioning.

- Sensor molecules located in the endoplasmic reticulum acting as mediators, maintain proteostasis under physiological and stressful conditions.

- In disease states when these inherent mechanisms are overwhelmed, misfolded proteins accumulate and the proapoptotic branch of the UPR is activated.

- Endoplasmic reticulum stress is implicated in numer- ous human diseases, including neuropsychiatric ailments.

- The finding that several chemicals including some newly discovered small non-toxic molecular compounds regulate ER - related processes has the promise of uncovering fresh avenues in the treatment of many recalcitrant neuropsychiatric disorders.

\section{INTRODUCTION}

Under both physiological conditions and cellular stress the unfolded protein response (UPR) embodies a conserved reaction ensuring protein quality control and cell survival. ${ }^{1}$ At the sub-cellular level the endoplasmic reticulum (ER) 
wields the UPR, as here, new proteins are synthesized, nascent polypeptides are given 3-D conformation, and posttranslational modifications take place. To perform its varied functions, the ER undertakes crucial interactions with other organelles, particularly mitochondria to regulate proteostasis and relegate misshapen proteins to degradation. In this regard, the ER and mitochondria lie in close apposition and share a significant portion of their membranes called 'mitochondria-associated membranes' or (MAM). In the case of UPR, mutant proteins are degraded via catabolic pathways characterized by the ubiquitin proteasome system and autophagic lysosomes. ${ }^{2} \mathrm{As}$ an integral function, the ER wields the unfolded protein response to protect the cell from toxicity of accumulated unfolded/misfolded proteins, whereas mitochondria also have their own protein quality control system, the UPR $(\mathrm{mt})^{3}$

ER buildup of unfolded proteins may be caused by such diverse factors as genetic defects in protein folding, posttranslational modifications, intracellular calcium alterations, redox imbalance, and inflammatory signaling. The UPR either results in reinstating cell homeostasis or consigning them to apoptosis; the adaptive processes are represented by ER associated biogenesis, the ubiquitin-proteasome system and autophagy. ${ }^{4}$ Fig. 1 schematically shows these compensatory mechanisms, whose failure

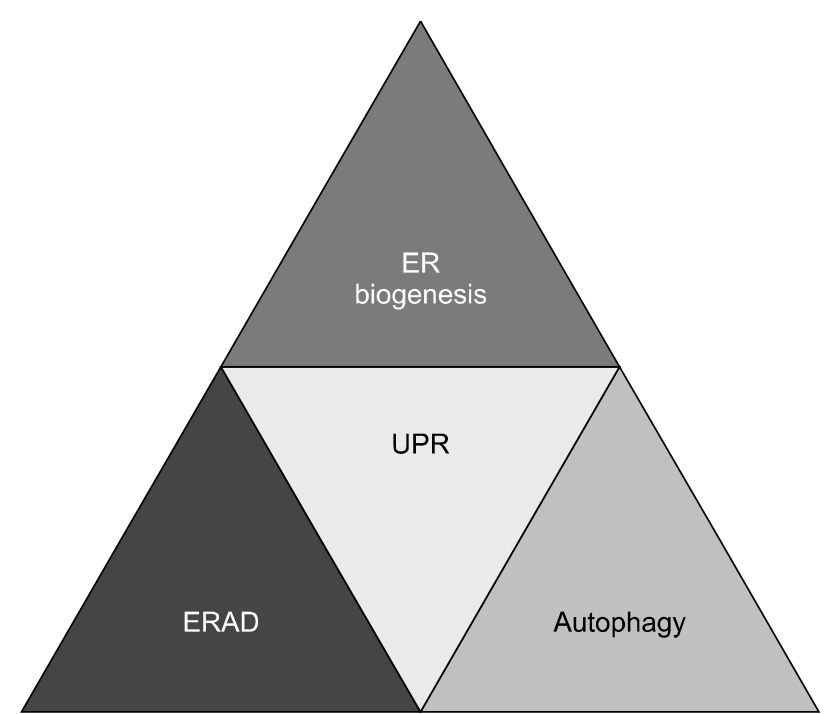

FIG. 1. Cellular adaptive response to endoplasmic reticulum (ER) stress. Increase in unfolded proteins can be instigated by physiological as well as disease factors. The latter may include defects in protein folding, calcium changes, redox imbalance and inflammatory signaling, resulting in the unfolded protein response (UPR). The cellular reaction to UPR involves other organelles, such as the mitochondria, and either leads to re-establishing cell homeostasis or relegating them to death. The cascades activated by UPR result in such adaptive reactions like ER associated biogenesis, ER-associated degradation (ERAD) via the ubiquitin-proteasome system and autophagy. When these regulating mechanisms are overwhelmed, stress sensors responsible for UPR commit cells to apoptosis. leads to the activation of the pro-apoptotic branch of the UPR and cell death (Fig. 1).

ER related stress mechanisms are incriminated in numerous human diseases including neuropsychiatric disorders, diabetes mellitus, cancer, cardiovascular, kidney, and pulmonary diseases. With respect to cellular resilience pathways, UPR signaling takes part in growth and development, and is also involved in proper brain functioning through long-term potentiation and neuronal plasticity processes. ${ }^{5}$

\section{ER STRESS SENSORS AND THE UPR-AN OVERVIEW}

The mediators of UPR are three bona fide effectors namely, inositol-requiring enzyme 1 (IRE1), activating transcription factor 6 (ATF6), and protein kinase RNA-like ER kinase (PERK) that regulate a multifaceted cascading pathway. ${ }^{6}$ Fig. 2 depicts in a simplified manner the canonical ER catalysts responsible for executing the intrinsic protein quality control system (Fig. 2).

Glucose related protein (GRP) 78, alternatively called

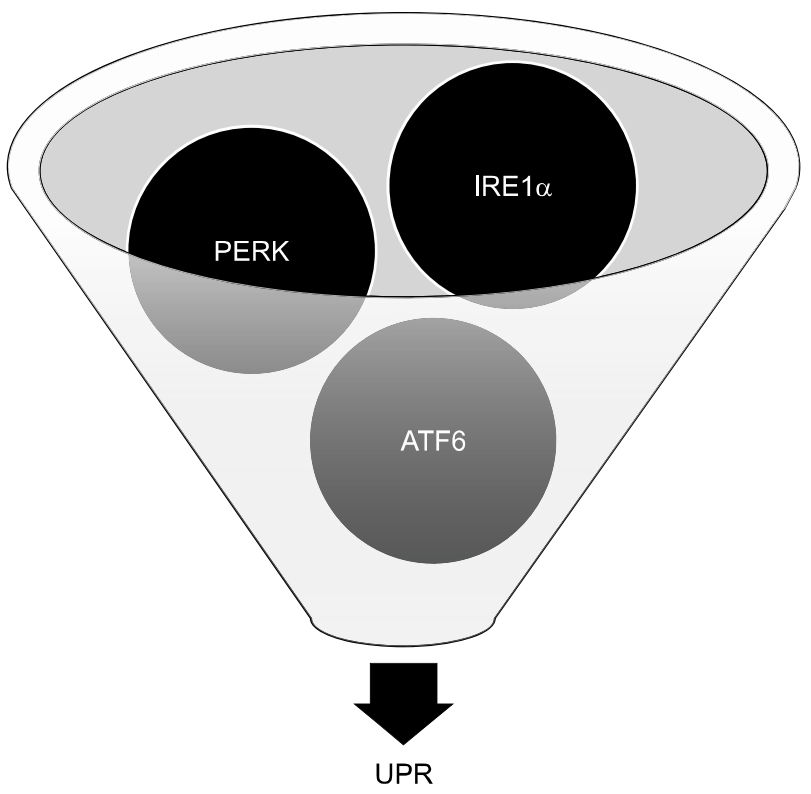

FIG. 2. Canonical mediators of the unfolded protein response (UPR). A third of all human proteins are folded into 3-dimensional conformations in the endoplasmic reticulum (ER) before they can perform their roles in cells. If a cell is required to make a large number of proteins, or in disease states unfolded proteins may accumulate in the ER. The organism responds to this stress by triggering the UPR which is mediated by three ER stress sensors-the inositol-requiring enzyme 1 (IRE1), the activating transcription factor 6 (ATF6) and protein kinase RNA-like ER kinase (PERK). These stimulate a complex transcriptional cascade with ensuing adaptive responses which restore the folding capabilities of the ER. However, if the ER stress exceeds the capacity of this built-in quality control, cell death is incited through the up-regulation of the pro-apoptotic arm of the UPR pathway to protect the organism from the toxicity of misfolded proteins. 
$\mathrm{BiP}$ is an ER chaperone, closely associated with the launching of UPR, and is among the best studied in this family of proteins. ${ }^{7}$ When the misshapen protein load increases in ER lumen, GRP78 dissociates from trans-membrane sensors kicking off the UPR. In this regard, ATF6 leads to the transcription of main ER chaperones and X-box binding protein-1 (XBP1). The endonuclease action of IRE1 catalyzes the splicing of $X B P 1 \mathrm{mRNA}$, causing the formation of an active transcription factor that regulates the expression of genes involved in the execution of UPR. The working together of ATF6 and IRE1-XBP1 facilitates the expression of ER chaperones, promotes the accurate configuration of the secreted proteins, prevents their accumulation, and enhances the continued existence of the cells. ${ }^{8}$ Conversely, when this compensatory mechanism is overwhelmed, the pro-apoptotic branch of the UPR is triggered by activated PERK; initially it dampens overall protein translation by phosphorylating the eukaryotic initiation factor $2 \alpha$ (eIF $2 \alpha)$ and decreasing the total amount of secreted proteins in the ER lumen. However, phosphorylated PERK also promotes the translation of some UPR-related genes including ATF4, inducing the activation of C/EBP homologous protein (CHOP). The later acts as a transcription factor that provokes cell death by directly inhibiting the expression of anti-apoptotic factor, Bcl-2. ${ }^{9}$ Fig. 3 provides an illustrated version of the main molecular events of the UPR in eukaryotic cells (Fig. 3).

\section{CANONICAL EFFECTORS OF UPR}

Membrane spanning catalysts in the ER act as sensing molecules and identify such perturbations as build-up of mutant proteins, variations in the levels of reactive oxygen species (ROS) and ionic calcium fluxes. IRE1 $\alpha$, PERK and ATF6, the three main mediators guaranteeing proteostasis, i.e. proper secretion, assembly and incorporation of translated proteins, ensure cell homeostasis. These are discussed in further detail below.

\section{Inositol-requiring enzyme 1 alpha (IRE1 $\alpha$ )}

IRE $1 \alpha$ has a reactive kinase region projecting in the cytosol and a luminal endoribonuclease domain; once activated by oligomerization and phosphorylation it plays a crucial role in protein quality control. Indeed, the endoribonuclease cuts out a $26 \mathrm{bp}$ intron from the pre-mRNA of $X B P-1$ resulting in the formation of a dynamic transcription factor. The latter has such major effects as up-regulation of ER chaperone genes and the modulation of ERAD. ${ }^{10}$ Thus, XBP-1 enhances the protein folding capacity of the ER and accelerates degradation of misfolded proteins, ensuring cell survival. New light is shed on the crystalline composition of yeast IRE1 as nascent peptides interact with its luminal projection and subsequently IRE1 oligomers are produced via self-association, which are observable as large structures in the ER. ${ }^{11}$ Demonstrated thus far in fungi, the eukaryotic IRE1 shows a somewhat similar configuration signifying a comparable type of control. ${ }^{12}$ This

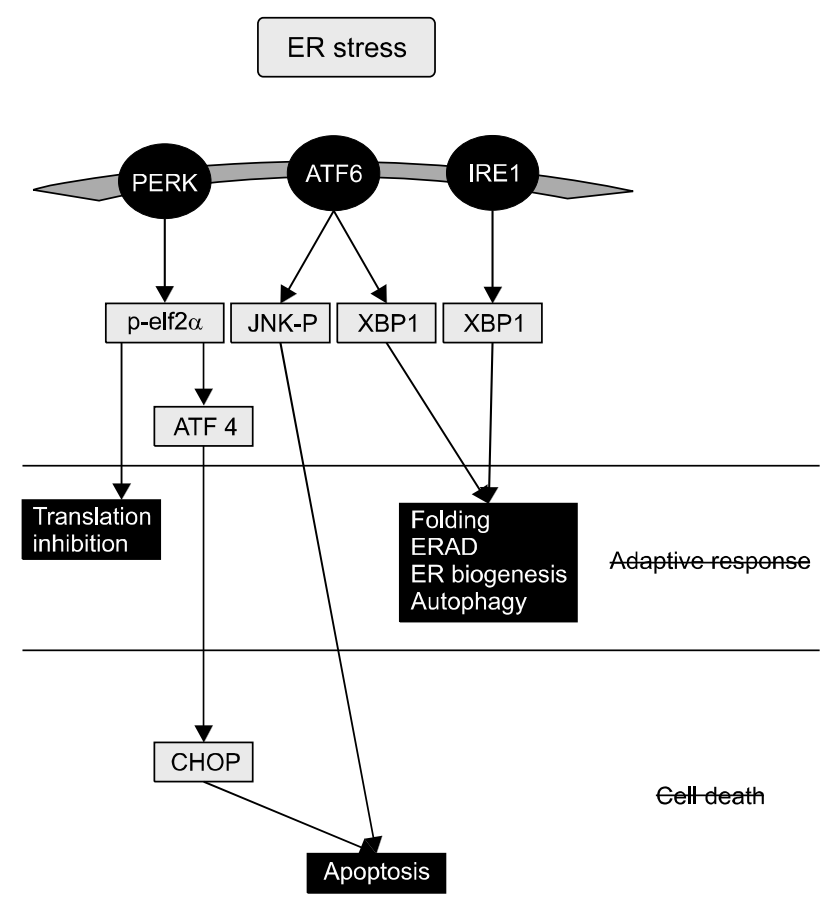

FIG. 3. Molecular mechanisms of endoplasmic reticulum stress response. Build-up of unfolded proteins in the ER lumen signals the unfolded protein response. The activated stress sensors - protein kinase RNA-like ER kinase (PERK), inositol-requiring enzyme 1 (IRE1) and activating transcription factor 6 (ATF6) - stimulate diverse cascades seeking to reinstate cell homeostasis or assign it to death. Here, the molecular events that occur in this process are explained in a simplified manner. In brief, IRE1 and ATF6 increase levels of XBP1 which is a diverse transcription factor and leads to increased expression of chaperones and other proteins involved in protein folding in ER. PERK, by virtue of phosphorylating elongation factor $2 \alpha$ (elf $2 \alpha$ ) puts a hold on translation, enabling ER to recover its protein folding capabilities. However, the expression of ATF4 is also increased which causes activation of $\mathrm{CHOP}$ and programmed cell death. The latter is also facilitated by c-Jun N-terminal kinase (JNK), a transcription factor stimulated by activated ATF6. CHOP: CCAAT/enhancer-binding protein homologous protein, ER: endoplasmic reticulum, ERAD: endoplasmic reticulum-associated protein degradation.

way of functioning of IRE1 is at disagreement with earlier conceptualizations which assumed that the binding of $\mathrm{BiP}$ by unfolded proteins was critical in triggering IRE1 signaling. Nevertheless, it is probable that BiP has a role in ensuring finer control of UPR so that its increased expression reduces ER stress and enhances cell survival.

\section{Protein kinase RNA-like endoplasmic reticulum kinase (PERK)}

The most important substrate of this enzyme is the eukaryotic translation initiation factor-2 $\alpha$ (eIF2 $\alpha$ ) whose phosphorylation causes an overall decrease in protein production and results in a reduction in the quantity of misfolded polypeptides. Nevertheless, the hold on translation 
is not absolute and certain key mediators, most significantly the transcription factor ATF4 are largely unaffected, and reciprocally activated. This has important downstream effects, for instance increased expression of the transcription factor CHOP, as well as modulation of the apoptosis regulating BCL-2 related molecules. GADD34 (growth arrest and DNA damage-inducible protein), also referred to as PPP1R15A (protein phosphatase 1 regulatory subunit 15A) acts as a phosphatase for p-IF2 $\alpha$ with the capacity to re-establish the translation of new proteins. It is up-regulated by certain transcription factors, not under the control of PERK. Hence, the degree of eIF2 $\alpha$ phosphorylation is dynamically regulated in the cell and can, therefore, be manipulated by employing precise activators or antagonists with the capacity to normalize ER stress in a range of pathological conditions. ${ }^{13}$ Table 1 gives an account of the recently identified small molecular compounds, targeting various components of the UPR machinery; these are non-toxic and can be administered orally and circumvent the need for genetic engineering (Table 1).
The ER stress sensors act in concert and their mutual interaction plays a pivotal role in determining cell fate. In this vein, an initial, temporary surge in IRE1 $\alpha$ activity instigates cell survival, while a preliminary PERK-ATF4 response leads to cell death. The reciprocal control of UPR signaling is illustrated by the divergent effects of the chief mediators on death receptor 5 (DR5) functioning. This is stimulated by the PERK-CHOP cascade, whereas the endonuclease activity of IRE1 promotes the cleavage of its mRNA. ${ }^{14}$ DR5 has a crucial role in controlling the proliferation of tumor cells, such that the UPR pathway, which has an overarching effect in determining the fate of cancerous lesions.

\section{Activating transcription factor 6 (ATF6)}

The activation of ATF6 is different from IRE1 $\alpha$ and PERK, as initially its translocation takes place from the ER to the Golgi apparatus. Site- 1 and site- 2 proteases, located within the membranes of the Golgi apparatus, catalyze the formation of active ATF6 by the process of proteolysis.

TABLE 1. Small molecules/compounds that target ER stress signaling in preclinical disease models

\begin{tabular}{|c|c|c|c|c|}
\hline Disease & Chemical & UPR signaling & Main effect & Comments \\
\hline $\mathrm{ALS} \alpha$ & Sephin 1 & $\begin{array}{l}\text { Inhibition of the stress- } \\
\text { induced holophosphatase } \\
\text { PPP1R15A }\end{array}$ & $\uparrow \mathrm{p}$-eIF2 $\alpha$, neuroprotection & $\begin{array}{l}\text { Shown to protect mice neurons from } \\
\text { otherwise lethal protein misfolding } \\
\text { stress }\end{array}$ \\
\hline $\begin{array}{l}\text { Ischemia } \\
\text { induced } \\
\text { glutamate } \\
\text { excitotoxicity }\end{array}$ & Salubrinal & $\begin{array}{l}\text { Inhibition of PP1, a } \\
\text { p-eIF } 2 \alpha \text { phosphatase }\end{array}$ & $\uparrow$ p-eIF2 $\alpha$, neuroprotection & $\begin{array}{l}\text { Salubrinal penetrates BBB and } \\
\text { activates PERK-ATF4 UPR pathway } \\
\text { enhancing the protein folding capacity } \\
\text { of the ER }\end{array}$ \\
\hline $\mathrm{PD}$ & Salubrinal & $\begin{array}{l}\text { Inhibition of } \\
\text { dephosphorylation of } \\
\text { p-eIF } 2 \alpha\end{array}$ & $\begin{array}{l}\text { Neuroprotection in } \alpha \text {-synuclein } \\
\text { mouse model }\end{array}$ & $\begin{array}{l}\text { Alpha-synuclein interacts with ER } \\
\text { chaperones and sensitizes neurons to } \\
\text { UPR-mediated toxicity. Salubrinal } \\
\text { reduces ER stress and attenuates } \\
\alpha \text {-synuclein dependent dopaminergic } \\
\text { neurodegeneration }\end{array}$ \\
\hline Prion disease & GSK2606414 & PERK inhibitor & $\begin{array}{l}\downarrow \text { p-eIF } 2 \alpha, \uparrow \text { in synaptic } \\
\text { proteins, } \downarrow \text { in prion mediated } \\
\text { neurodegeneration }\end{array}$ & $\begin{array}{l}\text { UPR activation and } \uparrow \text { p-eIF } 2 \alpha \text { are seen } \\
\text { in AD, PD and prion disease resulting } \\
\text { in transient shut down of protein } \\
\text { translation. An oral compound, } \\
\text { GSK2606414 has been shown to rescue } \\
\text { translational block in hippocampal } \\
\text { slices of prion infected mice }\end{array}$ \\
\hline $\begin{array}{l}\text { Drug } \\
\text { dependence }\end{array}$ & ISRIB & $\begin{array}{l}\text { Promotes guanine } \\
\text { nucleotide exchange } \\
\text { factor eIF2B activity }\end{array}$ & $\begin{array}{l}\text { Reverses translational block } \\
\text { induced by p-eIF2 } \alpha \text { and } \\
\text { increases cocaine induced LTP } \\
\text { in VTA dopaminergic neurons }\end{array}$ & $\begin{array}{l}\text { p-eIF2 } \alpha \text { levels control compulsive drug } \\
\text { taking behavior by regulating LTP in } \\
\text { the VTA. In a mouse model decreasing } \\
\text { its activity increased compulsive } \\
\text { cocaine taking }\end{array}$ \\
\hline $\begin{array}{l}\text { Cultured } \\
\text { cell-based } \\
\text { screening }\end{array}$ & $\begin{array}{c}\text { Compounds } \\
147 \& 263\end{array}$ & $\begin{array}{l}\text { Activate the endogenous } \\
\text { ATF } 6 \text { branch of UPR } \\
\text { independently of IRE1 } \\
\text { and PERK }\end{array}$ & $\begin{array}{l}\text { Selectively reduce the } \\
\text { extracellular secretion and } \\
\text { aggregation of misfolded } \\
\text { proteins responsible for } \\
\text { amyloid diseases }\end{array}$ & $\begin{array}{l}\text { Small non-toxic compounds that target } \\
\text { the ATF } 6 \text { arm of the UPR have the } \\
\text { promise to treat degenerative diseases } \\
\text { by ER re-programming. }\end{array}$ \\
\hline
\end{tabular}

AD: Alzheimer's disease, ALS: amyotrophic lateral sclerosis, ATF6: activation transcription factor 6, BBB: blood brain barrier, ER: endoplasmic reticulum, LTP: long term potentiation, PD: Parkinson's disease, PERK: protein kinase RNA-like ER kinase, p-eIF2 $\alpha$ : phosphorylated eukaryotic translation initiation factor 2 alpha, PP1: protein phosphatase 1, UPR: unfolded protein response, VTA: ventral tegmental area. 
ATF6 akin to IRE1 induces gene expression of ER chaperons, ERAD constituents and XBP- $1 .{ }^{15}$ In mammals, ATF6 expression is controlled by two correlated genes, ATF6 $\alpha$ and ATF6 $\beta$, and their vital role is illustrated by the fact that double deletion causes death in-utero in murine models. ATF6 is important for development, and experiments in medaka fish have been helpful in understanding this process. In this species, ER stress is involved in the embryogenesis of the brain and notochord, which is apparently brought about by the increased production of extracellular matrix (EM) proteins essential for organ formation. Experiments demonstrated that the deficiency of ATF6 caused a plummeting of $\mathrm{BiP}$ and other ER chaperone levels, retarded the folding and secretion of pro-collagen, and gravely impaired the formation of vital organs. ${ }^{16}$

\section{ER REPROGRAMIMING-AN AREA OF FURTHER STUDY}

During the process of protein quality control, UPR is involved in the extrusion to the extracellular space of proteins with a high propensity for aggregation. These polypeptides are often misfolded and accumulate in tissues as soluble fibrillary compounds, seen in a variety of diseases typified by amyloid build-up. Activated UPR can increase the level of ER chaperones, for example the protein ERdj3; it therefore controls misfolded, aggregate-prone protein secretion at the cell surface while coordinating this with the process of proteostasis inside the cell. ${ }^{17}$ Misfolded transthyretin (TTR) is an aggregate-prone protein, and ATF6 signaling is able to prevent amyloid formation and offset cytotoxicity by decreasing secretion of the mutant TTR. ${ }^{18}$ In a process called ER reprogramming, recent series of experiments identified small molecular compounds that emulate the functioning of ATF6 in diminishing the secretion of amyloid producing proteins. While serving as an important means of investigating ER stress, these original chemicals reduce the synthesis of misfolded polypeptides. Therefore, they are important in the future development of new therapeutic agents for human amyloidosis diseases, such as Alzheimer's disease. ${ }^{19}$ See also Table 1 for further information on these novel compounds.

\section{MITOCHONDRIAL UNFOLDED PROTEIN RESPONSE}

Mitochondria are vital for cell survival, and as with the ER have a protein quality control system exemplified by specific proteases. Unfolded protein response is a conserved response across species and UPR (mt) is as well studied in the worm Caenorhabditis elegans. In C. elegans, the activating transcription factor associated with stress-1 (ATFS-1) is a key regulator of UPR (mt) responsible for instigating nuclear transcription of mitochondrial chaperones and protective proteins. ${ }^{20}$ ATFS- 1 aids in cross-talk between mitochondria and nucleus; it is typically conveyed to the former, but under conditions of cellular stress the mi- tochondrial import mechanism is malfunctioning which increases ATFS-1 in the nucleus, causing expression of UPR (mt)-linked genes. Furthermore, polymorphisms in mitochondrial DNA (mt DNA) are connected to UPR (mt) and cellular senescence, even though this state of affairs is not so clear-cut. ${ }^{21}$ Accordingly, experiments in $C$. elegans show that UPR (mt) is intrinsically activated in heteroplasmic strains and this leads to soaring numbers of $\mathrm{mt}$ DNA deletions. ${ }^{22}$ It is an open question as to whether comparable mechanisms exist in mammalian cells, for instance in the aging process. Nevertheless, recent research demonstrates that activating transcription factor 5 (ATF5) performs an analogous function in mammalian mitochondrial stress as does ATFS-1 in C. elegans. ${ }^{23}$ In this regard, further studies are warranted to uncover functional connections of the ATF5-dependent signaling to those of mitochondrial degradation pathways activated in neurodegenerative disorders like Parkinson's disease.

Moreover, the ER and mitochondria are present in close proximity and share approximately $20 \%$ of their membranes. The MAM enables actual, physical transfer of stimuli between the two organelles and also facilitate chemical signaling $\left(\mathrm{Ca}^{++}, \mathrm{ROS}\right)$, an interaction that is gaining primacy in understanding processes vital for cell survival. In human degenerative diseases increased oxidative stress is present which entails mitochondrial formation of reactive oxygen species. Current research has shown that YME1L, a protease resident on the inner mitochondrial membrane is especially susceptible to ROS with the ensuing decreased capability for handling important mitochondrial proteins during cell stress. YME1L, together with the catalysts AFG3L2 and paraplegin, fits into the group of proteases with ATP binding sub-domains (ATP-dependant AAA+ proteases), responsible for protein quality control. ${ }^{24}$ Alterations or deficiencies in these effectors cause blighted mitochondrial function and result in such human pathologies as spinocerebellar ataxia type 28 and hereditary spastic paraplegia type $7 .^{25}$ Thus, UPR (mt) is associated with the development of various diseases, and in this regard diminished activity of mitochondrial proteases promotes cell stress by boosting the formation of protein aggregates.

\section{UNFOLDED PROTEIN RESPONSE IN NEUROPSYCHIATRIC DISORDERS}

Many human neurodegenerative disorders such as Alzheimer's disease (AD), Parkinson's disease (PD), amyotrophic lateral sclerosis (ALS), and Huntington's disease (HD) have distinctive pathologies typified by the build-up of misfolded proteins. Clearly, persistent ER stress is present in such conditions and this is verified in experiments which manipulate UPR signaling in laboratory disease models. Results signify that the role of UPR in these diverse conditions is multifaceted and depends on several factors such as the sequencing, potency and characteristics of a specific stressful event in disease development. ${ }^{26}$ 


\section{Unfolded protein response in $A D$ and prion diseases}

$\mathrm{AD}$ is characterized by the build-up of extracellular aggregates of amyloid- $\beta(\mathrm{A} \beta)$ oligomers and intracellular neurofibrillary tangles of the hyperphosphorylated tau protein. The cumulative effect of several derangements such as disrupted synaptic transmission, impaired calcium homeostasis, and unrelenting neuroinflammation leads to cellular degeneration in $\mathrm{AD} .^{27}$ In this regard, studies employing cell cultures have shown that the IRE1/XBP1 subdivision of UPR has dual function with respect to $\beta$-amyloid toxicity and is either beneficial as in Drosophila or harmful as in C. elegans. ${ }^{28}$ In the latter scenario c-jun N-terminal kinase (JNK) pathway is activated and leads to amplified assembly of beta amyloid that exacerbates ER stress. ${ }^{29}$ Contrarily, in an $\mathrm{AD}$ mouse model, dampening of PERK activity decreases the p-eIF2 $\alpha$ levels and ameliorates memory deficits. These experiments point to specific ER signaling aberrations and also that particular groups of neurons in discrete brain areas are affected in a time-specific manner in disease development. ${ }^{30}$

In an effort to delineate the toxicity of $\mathrm{A} \beta$ fibrils, investigators have studied the composition of these precipitates from other angles. For instance, the oligomers also have non-protein constituents like glycosaminoglycan (GAG) polysaccharides that may accelerate $\mathrm{A} \beta$ aggregation, and using heparin as a substitute in nuclear magnetic resonance spectroscopy has been helpful in defining their relative position in the deposits. Elucidating the molecular architecture of $\mathrm{A} \beta$ in this way has importance in designing chemicals which may block amyloid build-up in $\mathrm{AD}$. Remarkably, the biochemical structure of beta amyloid in $\mathrm{AD}$ brains is different from in vitro $\mathrm{A} \beta$; furthermore, it may even vary from patient to patient in diverse clinical sub-types. This warrants further investigation into the neuropathology of $\beta$-amyloid oligomers to fulfill the promise of better therapeutics for $\mathrm{AD} .^{31}$

Prion pathologies resulting from self-propagating prion proteins $(\mathrm{PrP})$ are uncommon neurodegenerative disorders exemplified by Creutzfeldt-Jakob disease in humans and bovine spongiform encephalopathy in animals. Misshapen PrP accumulates in brain tissue as amyloid oligomers resulting in spongiform changes and progressive death of neurons. Disease progression is from cell to cell during PrP replication and the self-associating property of the protein is central to cell damage. ${ }^{32}$ In prion diseases, specific sites are present in the mutant protein that promote accumulation, and interestingly, comparable domains have been discovered in other polypeptides like the malformed TAR DNA-binding protein 43 (TDP-43) that produces cytoplasmic inclusions in ALS. Prion pathologies increase our understanding of abnormal protein handling and propagation of transmittable particles in several neurodegenerative diseases. In this respect, new investigations inform us that a process akin to prion diseases may be to blame in the cellular accumulation of $\alpha$-synuclein in PD. $^{33}$

In mouse models of prion diseases, UPR and the PERK pathway are highly stimulated and a persistent rise in phospho-eIF2 $\alpha$ decreases the production of synaptic proteins leading to the loss of neurons. Contrarily, decreased activity of the PERK or increased expression of the GADD34 phosphatase reduces the level of p-eIF2 $\alpha$, resulting in improved synaptic functioning. This lends support to the assumption that manipulation of the PERK/p-eIF $2 \alpha$ pathway is a promising target for the discovery of novel medications in prion diseases. Current research in prioninfected mouse brain tissue has been helpful in delineating specific microRNAs and DNA segments that are linked to synaptic functioning. In addition to discovering peripheral disease markers for the timely diagnosis of prion diseases, these results may aid in identifying crucial pre-symptomatic phases in such pathologies. ${ }^{34}$

\section{UPR in PD, ALS and HD}

Protective functioning was linked to IRE1 $\alpha$ /XBP1 cascade, but intriguingly the UPR appeared to play a dual role in neurodegenerative conditions as a deficiency of XBP1 unexpectedly enhanced survival in a mouse model of ALS. Moreover, a lack of XBP1 increased autophagy via the forkhead box 01 (FOXO1) transcription factor, enhancing the catabolism of misfolded superoxide dismutase 1 (SOD1). ${ }^{35}$ In PD where dopaminergic neurons undergo degeneration, a lack of XBP1 produced dissimilar results according to the age of experimental mice which denoted corresponding alterations in UPR-controlled pathways. Accordingly, the absence of XBP1 during postnatal growth provoked ER stress which was physiological with defense against neuronal toxicity induced by 6 -hydroxydopamine, even as deletion of XBP1 in adulthood caused persistent ER stress and death of the dopaminergic neurons. ${ }^{36}$ Additionally, when salubrinal (an inhibitor of PPP1R15A/GADD34 phosphatase) was given to genetically engineered mice over expressing misfolded $\alpha$-synuclein , p-eIF $2 \alpha$ levels were increased, which was neuro-protective, signifying that the PERK pathway contributed to dopamine neuron degeneration. $^{37}$

In experimental paradigms where an acute neuronal injury was produced by lesions of the spinal cord, local delivery of XBP1 was beneficial, leading to the continued existence of cells and enhanced locomotion of animals. ${ }^{38}$ This implied that by fine-tuning IRE $1 \alpha / \mathrm{XBP} 1$ branch of the UPR, novel drugs could be discovered for application in diverse neurological conditions. Furthermore, XBP1 may also be implicated in crucial neuronal functions, including the formation of memories and new learning. Brain-derived neurotrophic factor (BDNF) is important in the vital processes regulating neuronal plasticity and long-term potentiation (LTP), and varying expression of XBP1 is incriminated in this function. LTP is also influenced by the phosphorylation state of eIF $2 \alpha$ which is controlled by PERK. These observations point to the significance of UPR signaling in memory-related processes, and further studies are needed to elucidate the findings. ${ }^{39}$

Amytrophic lateral sclerosis, a catastrophic disease, is 
characterized by the degeneration of $\alpha$-motoneurons in the spinal cord and corticospinal motor neurons (CSMNs) in the cerebral cortex. Experiments in transgenic mice have shown that the ubiquitin C-terminal hydrolase-L1 in the CSMNs is particularly vulnerable to ER stress which is induced by the deletion of the ALS-linked gene, Alsin. In this pathology, ER stress and autophagy contribute to the loss of CSMNs, hinting at promising new therapeutic targets. ${ }^{40}$ Furthermore, fresh studies have revealed that ALS and frontotemporal dementia are related and have in common a key genetic variation i.e. the existence of a hexanucleotide repeat sequence in the gene, C9orf72. Epigenetic modification of the gene function may provide a new understanding of the sub-cellular processes fundamental to neuronal dysfunction in these ailments. ${ }^{41}$ The phosphatase, PPP1R15A/GADD34 which regulates the levels of p-eIF2 $\alpha$ is an important therapeutic target in ALS. In this vein, it has been demonstrated that the chemical guanabenz is capable of prolonging eIF $2 \alpha$ phosphorylation and buttressing proteostasis. Nonetheless, guanabenz is an adrenergic agonist resulting in hypotension and consequently is of little utility in actual patients. Further testing was instrumental in the recognition of a novel compound, Sephin1, which selectively inhibited a holophosphatase capable of modulating the PERK/p-elf2 pathway and reinstated proteostasis. In experimental conditions in the altered SOD1-ALS mouse model, sephin1 showed neuro-protective properties, improved behavior, and had no significant adverse effects. Further, this molecule averted demyelination in the Charcot-Marie-Tooth disease model produced by the misfolded myelin protein zero. ${ }^{42}$ New research should demonstrate whether sephin 1 or analogous chemicals would be of value in ALS and other human protein misfolding disorders.

Investigations into prototypes of HD revealed that the silencing of XBP1 was neuro-protective by decreasing the aggregation of the misshapen Huntingtin protein (Htt) and promoting the existence of neurons. Similar to ALS, in HD, a lack of XBP1 increased ER related compensatory mechanisms like autophagy and up-regulated FOXO1 expression. Moreover, it has been revealed lately that the ubiquitin-specific protease 14 was instrumental in the catabolism of misfolded Htt by recruiting IRE1 $\alpha$ and modulating the proteasome function, further adding to the complexity of HD pathology. ${ }^{43}$ The accumulation of misfolded Htt differentially occurred in specific cellular areas like the cytoplasm and the nucleus, and lately it has been demonstrated that Htt-polymers were found along the nuclear membrane, possibly interfering with the physiological transport of transcription factors and mRNA. Additionally, expression of TDP-43, which is related to the development of ALS, also influenced this mechanism. ${ }^{44}$ This implies that nucleocytoplasmic variations in HD and other human protein misfolding pathologies need further investigation.

\section{Unfolded protein response in brain ischemia and epi- lepsy}

Following brain ischemia and stroke, the levels of the excitatory amino acid glutamate are increased leading to excitotoxicity, injury, and death of neurons. Acting through specific receptor types, glutamate increases intracellular calcium and decides neuronal fate. Enhanced ER stress is linked to hypoxic damage since in experimental paradigms where brain ischemia is induced by cerebral vessel occlusion, hypoxic injury and glutamate toxicity is stalled by Salubrinal which acts as a specific inhibitor of the eIF2 $\alpha$ phosphatase, incriminating UPR signaling in stroke models. $^{45}$

In epilepsy there is increased electrical activity in the brain leading to recurrent, aberrant neuronal discharges and seizures. The characteristic of convulsive activity differs among patients, and in the majority is controllable by medications. However, chronic temporal lobe epilepsy is often treatment refractory causing ER stress and particularly stimulating the IRE1 $\alpha$ pathway, adding to injury. ${ }^{46}$ This proposes that the mitigation of ER stress is of possible value in reducing damage to neurons in epilepsy, warranting further exploration of this avenue.

\section{UPR in major psychiatric disorders}

Admittedly, psychiatric disorder studies on the role of ER stress and UPR activation are in short supply. Bipolar disorder (BD) is a major mood disorder exemplified by episodes of depression and mania; its neurobiology is multifaceted and linked to other mental and physical disorders. Studies employing BD patient-derived blood cells revealed that the response to ER stress activating compounds like tunicamycin or thapsigargin was abnormal compared with controls suggesting compromised cellular reaction to stress. Lithium is the prototype mood stabilizer widely used in the management of $\mathrm{BD}$ and recent data has demonstrated that this medication is involved in the expression of genes which contribute to maintaining ER functioning. Together, this supports the premise that ER stress and UPR processes are important targets in the treatment of BD. ${ }^{47}$ Moreover, found within the ER and acting together with mitochondria is the sigma-1 receptor (Sig-1R) which is targeted by a range of psychotropic medications. A MAM residing chaperone, the Sig-1R is a key mediator and plays an important role under stressful conditions by modulating inter-organelle $\mathrm{Ca}^{++}$signaling. It facilitates the passage of stress signals from the ER to the nucleus via numerous receptors, ion channels, kinases, and regulatory proteins present in the ER, MAM, cytosol, and nucleus. Thus, this diverse regulator modulates bioenergetics, redox balance, UPR, and cytokine signaling. In the neurons, it partakes in dendrite outgrowth, synaptogenesis, and LTP and appears to play a vital role in neuropsychiatric diseases; however, this awaits further delineation. ${ }^{48}$

In $\mathrm{BD}$, a number of studies utilizing patient-derived lymphoblastoid cell lines or peripheral leucocytes have revealed anomalous reactions by the ER to induced stress. 
In a seminal study in BD patients' B-lymphocytes, So et al. ${ }^{49}$ reported lowered expression of XBP1 and CHOP genes when stimulated by thapsigargin and tunicamycin. These observations were strengthened by another study which found an increase in several proteins concerned in UPR (p-eIF2 $\alpha$, GRP78, XBP1 and CHOP) in leucocytes treated with tunicamycin from controls, but not in those from bipolar cases. There was more apoptosis of cells in cases compared to controls, and this phenomenon was greater in chronic patients, signifying that disease progression increased vulnerability to ER stress. ${ }^{50}$ In their study, Hayashi et al. ${ }^{51}$ treated BD patient-derived lymphoblastoid cell lines with thapsigargin and compared these to controls, showing a reduction in the expression of XBP1 and GRP94 in these cases. A mutation caused by a single nucleotide polymorphism (SNP; - 116C/G; rs2269577) in the promoter region of the XBP1 gene deregulated feedback loop in the ER response and was found to be linked to $\mathrm{BD} .^{52} \mathrm{An}$ interesting study examined RNA sequences in peripheral circulating leucocytes in healthy controls, lithium-responsive cases, and lithium-non-responsive patients. Discrepant gene analysis has showed that in $\mathrm{BD}$, the clinical response to lithium is modulated by a gene network that regulates ER stress. ${ }^{53}$ Generally, the above findings implied that in $\mathrm{BD}$, the compensatory reaction of cells to ER stress was blighted and this limited their survival under stressful conditions. Interestingly, the most commonly employed mood stabilizers, lithium and valproate, appeared to possess protective properties, since these enhanced the resilience of cells to ER stress. ${ }^{54}$

Autophagy represents a cellular process intended to re-establish homeostasis or assign cells to degradation in the wake of stressful events. In the course of autophagy, misfolded protein aggregates, membranous elements, and sub-cellular organelles are degraded and the resulting molecular compounds are reprocessed. Two pivotal cascades controlling autophagy-related genes are the phosphatidylinositol 3-kinase which is activated, along with the simultaneous inhibition of mTOR kinase. An intricate relationship is at work governing autophagy, ER stress, and mitochondrial function. In this respect, the effectors of UPR trigger autophagy and influence mitochondrial function by modulating the actions of Parkin. On the flip side, Parkin is a controller of mitochondrial function and is required to destine non-functioning mitochondria to mitophagy. ${ }^{55}$ Recently, a study observed that autophagy was decreased in schizophrenia and MDD. ${ }^{56}$ In this vein, a recently published paper conjectured that in psychiatric disorders, dysfunctions in cell biology were found, however, abnormalities in autophagy and mitochondrial functioning were of crucial significance. $^{57}$

\section{A CELLULAR PATHOGENIC MODEL OF MAJOR PSYCHIATRIC DISORDERS}

In the ongoing discussion regarding the most pertinent aspects of cellular functioning under pathological con- ditions are described and the pivotal role of the endoplasmic reticulum is highlighted. The mitochondrial operations are regulated through the lipid raft like ER membranes, or MAM, and these, in addition to controlling mitochondrial shape and motility, influence bioenergetics and redox balance. Besides, the MAM plays a critical role in cellular adaptation and survival via modulating ER stress, proteostasis, inflammasome signaling, and apoptosis. The ER associated transcription factors, chaperones, and catalysts have a pivotal function in this process, with the failure of the unfolded protein response causing cellular loss through accumulation of toxic protein aggregates. Miscommunication between vital cellular organelles likely underlies not just neuropsychiatric disorders, but also disorders of aging, metabolic conditions, and cancer. With respect to the development of principal psychiatric disorders a supposed cascade of events is as follows:

a) Severe or persistent ER stress is linked to increased ROS and $\mathrm{Ca}^{++}$.

b) Up-regulation of transcription factors (CHOP) and kinases (JNK) via activation of the pro-apoptotic branch of the UPR.

c) Failure of UPR leads to the accumulation of protein aggregates.

d) The latter cause stimulation of the inflammasome and increased inflammatory signaling through pro-inflammatory cytokines.

e) Activation of proapoptotic caspases with enhanced apoptosis of neurons.

Fig. 4 schematically depicts this likely model of disease progression while identifying new targets for the development of novel therapeutic agents (Fig. 4).

\section{CONCLUSION}

To reduce the load of unfolded or misfolded proteins and prevent abnormal protein aggregation, proteostasis entails dynamic coordination between the efficient folding of newly synthesized proteins, quality control, and degradation mechanisms. Chaperones are an essential first line defense in this process since they recognize non-native conformations of polypeptides, which are then consigned to refolding through the UPR. In the instance of a failure of this protective process, degradation mechanisms - ubiquitin proteasome pathway and autophagy are activated. Complementary in their mode of action, these pathways act as a subsequent step to restore proteostasis. When the proteasome is defective or saturated, or if there is an excess of ROS, the accumulation of misfolded proteins occurs leading to the formation of both toxic soluble oligomers and larger aggregates that can then be eliminated by autophagy. However, in several neuropsychiatric disorders, autophagic vacuoles (autophagosomes or autophagolysosomes) accumulate suggesting lysosomal dysfunction. Due to the impairment of the autophagic pathway, misfolded proteins and aggregates are not cleared from the cells resulting in cell death. 


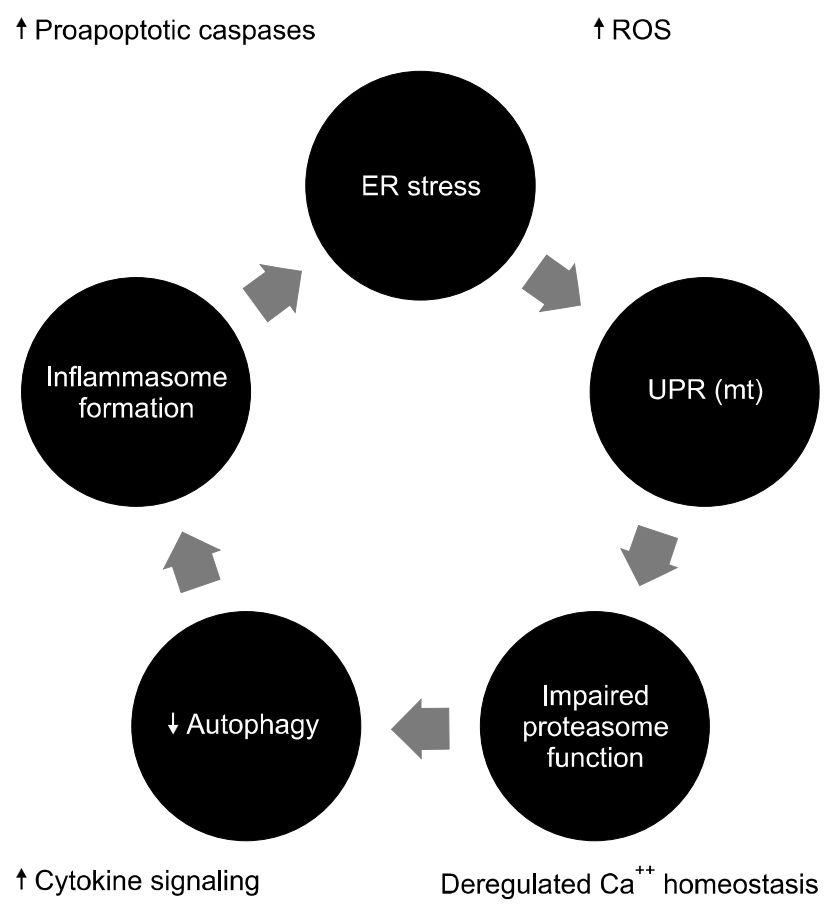

FiG. 4. A cellular pathogenic model of disease development in major psychiatric disorders. Major psychiatric disorders including mood disorders, psychotic disorders and neurodegenerative disorders are characterized by increased endoplasmic reticulum (ER) stress. ER chaperones act as first line of defense against unfolded or misfolded proteins, but accumulation of non-natively conformed polypeptides in the ER lumen activates other compensatory mechanisms. ER and mitochondria work in close unison through the mitochondria-associated ER membranes, stimulating the mitochondrial unfolded protein response (UPR $\mathrm{mt}$ ). However, in cellular stress increased generation of reactive oxygen species (ROS) and build up of intracellular calcium ions further exacerbates ER/mt stress. During proteostasis, unfolded/ misfolded proteins are degraded via the ubiquitin-proteasome system but when the latter gets saturated, toxic oligomers or larger aggregates accumulate, which can then be got rid of through lysosomal autophagic mechanisms. Since lysosomal dysfunction is present in many neuropsychiatric diseases, accumulated protein aggregates acting as danger associated molecular patterns cause activation of the inflammasome leading to inflammatoryimmune signaling and ultimately increased apoptosis of neurons. This cascade is responsible for increased damage to neurons and its further elucidation can result in the development of novel therapeutic agents for otherwise recalcitrant neuropsychiatric diseases.

The ER, a vital sub-cellular component, acts in concert with other organelles, most importantly the mitochondria through the MAM and this interaction either leads to cell survival or apoptosis. Indeed, crucial physiological processes including regulation of the metabolism, inflammation, neuronal plasticity, cognition, and aging are all modulated in an intricate manner. Therefore, it comes as no surprise that numerous human diseases including neuropsychiatric disorders are caused by dysfunction in the essential processes alluded to above. This understanding has lead to the development of specific compounds which target in- dividual branches of UPR and some of the recently identified chemicals with specific reference to CNS are summarized in Table 1, with the caveat that these small non-toxic molecules have been studied in preclinical models. Moreover, this catalog is essentially interim as many studies are in progresss and are yet to be published, signifying that novel compounds possessing the ability to fine tune the function of the ER will likely be added to this list. Targeting the MAM and the processes that involve protein degradation pathways and the inflammasome are valid options in the development of new therapeutic agents for essentially recalcitrant human diseases.

In summary, the ER stress response is closely associated with mitochondrial functioning; the failure of proteostasis leads to the formation of protein aggregates that induce an immune response through intracellular inflammasome formation, most importantly the NLRP3 inflammasome. The signals that provoke aberrant cellular responses include the buildup of ROS, abnormal $\mathrm{Ca}^{++}$ionic fluxes and pro-inflammatory cytokines. Under pathological conditions, compensatory homeostatic mechanisms fail and pro-apoptotic caspases are triggered with increased loss of cells. A better understanding of these complicated mechanisms is essential to find cures for numerous human diseases, including neuropsychiatric disorders.

\section{FUTURE PERSPECTIVE}

As evidence accumulates, it is becoming clear that UPR (ER) and UPR (mt) play a pivotal role in the advancement of numerous human diseases including neuropsychiatric ailments, such that targeting this pathway is a primary objective in the development of novel therapeutic agents. In this regard, the modulation of the ER - related quality control mechanisms via activation of protective and adaptive UPR responses, and/or the inhibition of the apoptotic pathways emerge as significant strategies. ${ }^{58}$ Drawing on chemical chaperones such as 4-phenylbutyric acid (PBA), tauroursodeoxycholic acid (TUDCA) or trimethylamine oxide (TMAO) may be beneficial as these compounds improve ER folding capacity and stabilize protein conformation. ${ }^{59}$ In an $\mathrm{AD}$ mouse model, PBA was shown to enhance ER functioning, thwart $A \beta$ accumulation, and avoid the loss of dendritic spines and memory. ${ }^{60}$ TUDCA, a taurine-conjugated derivative from ursodeoxycholic acid was found to prevent $A \beta$ production in the cortex and hippocampus of APP/PS1 mice through the regulation of APP processing, salvaging neurons, and improving memory. ${ }^{61}$ Although experimented upon less often, the chemical chaperone TMAO has been found to be more efficient than PBA and TUDCA in enhancing protein folding, decreasing buildup of protein aggregates, and preventing apoptosis; hence it can be considered a promising therapeutic strategy for protein misfolding disorders. ${ }^{62}$

$\mathrm{Ca}^{++}$is a vital player in inter-organelle communication through the MAM and many catalysts/chaperones do not work properly when $\mathrm{Ca}^{2+}$ homeostasis is blighted. Targeting 
$\mathrm{ER} \mathrm{Ca}^{2+}$ may allow the restoration of ER homeostasis and increase protein folding. The ryanodine receptor antagonist dantrolene, approved for the treatment of spasticity, inhibits $\mathrm{ER} \mathrm{Ca}^{2+}$ release and the activation of PERK, eIF2 $\alpha$ and CHOP/GADD153. In an AD mouse model, dantrolene reduced memory deficits and the burden of neuritic plaques in the hippocampus, while in primary cultures of cortical neurons, this compound partially prevented neuronal death induced by $\mathrm{A} \beta{ }^{63}$ Moreover, modulation of calcium signaling, for instance by xestospongin $\mathrm{C}$ (an inositol 3 phosphate receptor antagonist) is another avenue with potential benefits in protein misfolding disorders, at least in experimental paradigms. ${ }^{64}$

Chemicals that directly interact with UPR intermediaries can also be important therapeutic mediators. Salubrinal (3-phenyl- $N$-[2,2,2-trichloro-1-[[(8-quinolinylamino) thioxomethyl] amino] ethyl]-2-propen amide) was reported to inhibit the IRE1 $\alpha /$ JNK signaling pathway and also the GADD34-phosphatase complex that dephosphorylated eIF2 $\alpha$. Studies performed in primary cortical or hippocampal cultures and in human neuroblatoma cell lines treated with Salubrinal, observed an increase in phosphorylated eIF $2 \alpha($ peIF $2 \alpha)$ and an attenuation of ER stress-mediated apoptosis. ${ }^{65,66}$ A new screening discovered a selective inducer of GRP78/BiP, named BiP inducer X or BIX (1-(3,4-dihydroxy-phenyl)-2-thiocyanato-ethanone), that worked by stimulating the canonical pathway and was capable of up-regulating GRP78/BiP in an ATF6-dependent manner, but an IRE1- and PERK-independent manner. In another study BIX diminished the expression of CHOP/ GADD153 and averted cell death in mouse retina. ${ }^{67}$

A number of studies demonstrated that antioxidant compounds such as edaravone (3-methyl-1-phenyl-2-pyrazolin5 -one), dibenzoylmethane derivatives, and $N$-acetyl cysteine (NAC) had advantageous effects under conditions of ER stress. Edaravone was a free radical scavenger that salvaged neurons in several models of protein folding diseases, for example, in SH-SY5Y cells it checked A $\beta$ production through stimulation of the non-amyloidogenic APP processing. ${ }^{68}$ In primary cultures of glia, edaravone attenuated ER stress induced by tunicamycin and reduced cell death through the inhibition of CHOP/GADD153 and XBP1. Edaravone was also described as an inhibitor of PERK, eIF2 $\alpha$, ATF4 and caspase- $12 .{ }^{69} \mathrm{NAC}$ is a useful anti-oxidant that showed salutary effects during ER stress-mediated apoptosis as proposed by a study showing that it modulated several mediators of UPR such as phospho-PERK, CHOP/GADD153, ATF4, GADD34, GRP78/BiP, while splicing XBP1 in a transfected B-cell model. ${ }^{70}$ As a final note it must be stated that ER-related mechanisms have huge therapeutic potential, and harnessing these can result in cures for numerous intractable human ailments.

\section{CONFLICT OF INTEREST STATEMENT}

None declared.

\section{REFERENCES}

1. Fu XL, Gao DS. Endoplasmic reticulum proteins quality control and the unfolded protein response: the regulative mechanism of organisms against stress injuries. Biofactors 2014;40:569-85.

2. Jovaisaite V, Mouchiroud L, Auwerx J. The mitochondrial unfolded protein response, a conserved stress response pathway with implications in health and disease. J Exp Biol 2014;217: 137-43.

3. van Oosten-Hawle P, Morimoto RI. Transcellular chaperone signaling: an organismal strategy for integrated cell stress responses. J Exp Biol 2014;217:129-36.

4. Inagi R, Ishimoto Y, Nangaku M. Proteostasis in endoplasmic reticulum--new mechanisms in kidney disease. Nat Rev Nephrol 2014;10:369-78.

5. Freeman OJ, Mallucci GR. The UPR and synaptic dysfunction in neurodegeneration. Brain Res 2016;1648:530-7.

6. Scheper W, Hoozemans JJ. The unfolded protein response in neurodegenerative diseases: a neuropathological perspective. Acta Neuropathol 2015;130:315-31.

7. Zhu G, Lee AS. Role of the unfolded protein response, GRP78 and GRP94 in organ homeostasis. J Cell Physiol 2015;230:1413-20.

8. Jiang D, Niwa M, Koong AC. Targeting the IRE1 $\alpha$-XBP1 branch of the unfolded protein response in human diseases. Semin Cancer Biol 2015;33:48-56.

9. Galehdar Z, Swan P, Fuerth B, Callaghan SM, Park DS, Cregan SP. Neuronal apoptosis induced by endoplasmic reticulum stress is regulated by ATF4-CHOP-mediated induction of the Bcl-2 homology 3-only member PUMA. J Neurosci 2010;30:16938-48.

10. Jung J, Michalak M, Agellon LB. Endoplasmic reticulum malfunction in the nervous system. Front Neurosci 2017;11:220.

11. Concha NO, Smallwood A, Bonnette W, Totoritis R, Zhang G, Federowicz K, et al. Long-range inhibitor-induced conformational regulation of human IRE1 $\alpha$ endoribonuclease activity. Mol Pharmacol 2015;88:1011-23.

12. Yang J, Liu H, Li L, Liu H, Shi W, Yuan X, et al. Structural insights into IRE1 functions in the unfolded protein response. Curr Med Chem 2016;23:4706-16.

13. Trinh MA, Kaphzan H, Wek RC, Pierre P, Cavener DR, Klann E. Brain-specific disruption of the eIF2 $\alpha$ kinase PERK decreases ATF4 expression and impairs behavioral flexibility. Cell Rep 2012;1:676-88.

14. Lu M, Lawrence DA, Marsters S, Acosta-Alvear D, Kimmig P, Mendez AS, et al. Opposing unfolded-protein-response signals converge on death receptor 5 to control apoptosis. Science 2014;345:98-101.

15. Credle JJ, Forcelli PA, Delannoy M, Oaks AW, Permaul E, Berry DL, et al. $\alpha$-Synuclein-mediated inhibition of ATF6 processing into COPII vesicles disrupts UPR signaling in Parkinson's disease. Neurobiol Dis 2015;76:112-25.

16. Ishikawa T, Okada T, Ishikawa-Fujiwara T, Todo T, Kamei Y, Shigenobu S, et al. ATF6 $\alpha / \beta$-mediated adjustment of ER chaperone levels is essential for development of the notochord in medaka fish. Mol Biol Cell 2013;24:1387-95.

17. Genereux JC, Qu S, Zhou M, Ryno LM, Wang S, Shoulders MD, et al. Unfolded protein response-induced ERdj3 secretion links ER stress to extracellular proteostasis. EMBO J 2015;34:4-19. 
18. Chen JJ, Genereux JC, Qu S, Hulleman JD, Shoulders MD, Wiseman RL. ATF6 activation reduces the secretion and extracellular aggregation of destabilized variants of an amyloidogenic protein. Chem Biol 2014;21:1564-74.

19. Plate L, Cooley CB, Chen JJ, Paxman RJ, Gallagher CM, Madoux $\mathrm{F}$, et al. Small molecule proteostasis regulators that reprogram the ER to reduce extracellular protein aggregation. Elife 2016;5: e15550.

20. Arnould T, Michel S, Renard P. Mitochondria retrograde signaling and the UPR mt: where are we in mammals? Int J Mol Sci 2015;16:18224-51.

21. Schulz AM, Haynes CM. UPR(mt)-mediated cytoprotection and organismal aging. Biochim Biophys Acta 2015;1847:1448-56.

22. Nargund AM, Pellegrino MW, Fiorese CJ, Baker BM, Haynes CM. Mitochondrial import efficiency of ATFS-1 regulates mitochondrial UPR activation. Science 2012;337:587-90.

23. Fiorese CJ, Schulz AM, Lin YF, Rosin N, Pellegrino MW, Haynes CM. The transcription factor ATF5 mediates a mammalian mitochondrial UPR. Curr Biol 2016;26:2037-43.

24. Rainbolt TK, Saunders JM, Wiseman RL. YME1L degradation reduces mitochondrial proteolytic capacity during oxidative stress. EMBO Rep 2015;16:97-106.

25. Gorman GS, Pfeffer G, Griffin H, Blakely EL, Kurzawa-Akanbi M, Gabriel J, et al. Clonal expansion of secondary mitochondrial DNA deletions associated with spinocerebellar ataxia type 28 . JAMA Neurol 2015;72:106-11.

26. Milisav I, Šuput D, Ribarič S. Unfolded protein response and macroautophagy in Alzheimer's, Parkinson's and prion diseases. Molecules 2015;20:22718-56.

27. Heneka MT, Carson MJ, El Khoury J, Landreth GE, Brosseron F, Feinstein DL, et al. Neuroinflammation in Alzheimer's disease. Lancet Neurol 2015;14:388-405.

28. Duran-Aniotz C, Cornejo VH, Espinoza S, Ardiles ÁO, Medinas DB, Salazar C, et al. IRE1 signaling exacerbates Alzheimer's disease pathogenesis. Acta Neuropathol 2017;134:489-506.

29. Gourmaud S, Paquet C, Dumurgier J, Pace C, Bouras C, Gray F, et al. Increased levels of cerebrospinal fluid JNK3 associated with amyloid pathology: links to cognitive decline. J Psychiatry Neurosci 2015;40:151-61.

30. Rozpedek W, Markiewicz L, Diehl JA, Pytel D, Majsterek I. Unfolded protein response and PERK kinase as a new therapeutic target in the pathogenesis of Alzheimer's disease. Curr Med Chem 2015;22:3169-84.

31. Wang P, Ding K. Proteoglycans and glycosaminoglycans in misfolded proteins formation in Alzheimer's disease. Protein Pept Lett 2014;21:1048-56.

32. Genereux JC, Wiseman RL. Regulating extracellular proteostasis capacity through the unfolded protein response. Prion 2015;9:10-21.

33. Chen JJ, Genereux JC, Wiseman RL. Endoplasmic reticulum quality control and systemic amyloid disease: impacting protein stability from the inside out. IUBMB Life 2015;67:404-13.

34. Halliday M, Radford H, Mallucci GR. Prions: generation and spread versus neurotoxicity. J Biol Chem 2014;289:19862-8.

35. Riboldi G, Nizzardo M, Simone C, Falcone M, Bresolin N, Comi GP, et al. ALS genetic modifiers that increase survival of SOD1 mice and are suitable for therapeutic development. Prog Neurobiol
2011;95:133-48.

36. Valdés P, Mercado G, Vidal RL, Molina C, Parsons G, Court FA, et al. Control of dopaminergic neuron survival by the unfolded protein response transcription factor XBP1. Proc Natl Acad Sci U S A 2014;111:6804-9.

37. Colla E, Jensen PH, Pletnikova O, Troncoso JC, Glabe C, Lee MK. Accumulation of toxic $\alpha$-synuclein oligomer within endoplasmic reticulum occurs in $\alpha$-synucleinopathy in vivo. J Neurosci 2012; 32:3301-5.

38. Valenzuela V, Collyer E, Armentano D, Parsons GB, Court FA, Hetz C. Activation of the unfolded protein response enhances motor recovery after spinal cord injury. Cell Death Dis 2012;3:e272.

39. Martínez G, Vidal RL, Mardones P, Serrano FG, Ardiles AO, Wirth C, et al. Regulation of memory formation by the transcription factor XBP1. Cell Rep 2016;14:1382-94.

40. Gautam M, Jara JH, Sekerkova G, Yasvoina MV, Martina M, Özdinler PH. Absence of alsin function leads to corticospinal motor neuron vulnerability via novel disease mechanisms. Hum Mol Genet 2016;25:1074-87.

41. Liu EY, Russ J, Wu K, Neal D, Suh E, McNally AG, et al. C9orf72 hypermethylation protects against repeat expansion-associated pathology in ALS/FTD. Acta Neuropathol 2014;128:525-41.

42. Carrara M, Sigurdardottir A, Bertolotti A. Decoding the selectivity of eIF2 $\alpha$ holophosphatases and PPP1R15A inhibitors. Nat Struct Mol Biol 2017;24:708-16.

43. Hyrskyluoto A, Bruelle C, Lundh SH, Do HT, Kivinen J, Rappou $\mathrm{E}$, et al. Ubiquitin-specific protease-14 reduces cellular aggregates and protects against mutant huntingtin-induced cell degeneration: involvement of the proteasome and ER stress-activated kinase IRE1 $\alpha$. Hum Mol Genet 2014;23:5928-39.

44. Ederle H, Dormann D. TDP-43 and FUS en route from the nucleus to the cytoplasm. FEBS Lett 2017;591:1489-507.

45. Anuncibay-Soto B, Pérez-Rodríguez D, Santos-Galdiano M, Font E, Regueiro-Purriños M, Fernández-López A. Post-ischemic salubrinal treatment results in a neuroprotective role in global cerebral ischemia. J Neurochem 2016;138:295-306.

46. Liu GL, Wang KY, Guo H, Zhao SJ, Shen Y, Zhao YB. Inositol-requiring protein $1 \alpha$ signaling pathway is activated in the temporal cortex of patients with mesial temporal lobe epilepsy. Neurol Sci 2013;34:357-64.

47. Bengesser SA, Fuchs R, Lackner N, Birner A, Reininghaus B, Meier-Allard N, et al. Endoplasmic reticulum stress and bipolar disorder - almost forgotten therapeutic drug targets in the unfolded protein response pathway revisited. CNS Neurol Disord Drug Targets 2016;15:403-13.

48. Tsai SY, Pokrass MJ, Klauer NR, De Credico NE, Su TP. Sigma-1 receptor chaperones in neurodegenerative and psychiatric disorders. Expert Opin Ther Targets 2014;18:1461-76.

49. So J, Warsh JJ, Li PP. Impaired endoplasmic reticulum stress response in B-lymphoblasts from patients with bipolar-I disorder. Biol Psychiatry 2007;62:141-7.

50. Pfaffenseller B, Wollenhaupt-Aguiar B, Fries GR, Colpo GD, Burque RK, Bristot G, et al. Impaired endoplasmic reticulum stress response in bipolar disorder: cellular evidence of illness progression. Int J Neuropsychopharmacol 2014;17:1453-63.

51. Hayashi A, Kasahara T, Kametani M, Toyota T, Yoshikawa T, Kato T. Aberrant endoplasmic reticulum stress response in lym- 
phoblastoid cells from patients with bipolar disorder. Int $\mathrm{J}$ Neuropsychopharmacol 2009;12:33-43.

52. Cheng D, Zhang K, Zhen G, Xue Z. The -116C/G polymorphism in XBP1 gene is associated with psychiatric illness in Asian population: a meta-analysis. Am J Med Genet B Neuropsychiatr Genet 2014;165B:665-72.

53. Breen MS, White CH, Shekhtman T, Lin K, Looney D, Woelk CH, et al. Lithium-responsive genes and gene networks in bipolar disorder patient-derived lymphoblastoid cell lines. Pharmacogenomics J 2016;16:446-53.

54. Kakiuchi C, Ishigaki S, Oslowski CM, Fonseca SG, Kato T, Urano F. Valproate, a mood stabilizer, induces WFS1 expression and modulates its interaction with ER stress protein GRP94. PLoS One 2009;4:e4134.

55. Durcan TM, Fon EA. The three 'P's of mitophagy: PARKIN, PINK1, and post-translational modifications. Genes Dev 2015; 29:989-99.

56. Merenlender-Wagner A, Malishkevich A, Shemer Z, Udawela M, Gibbons A, Scarr E, et al. Autophagy has a key role in the pathophysiology of schizophrenia. Mol Psychiatry 2015;20:126-32.

57. Toker L, Agam G. Mitochondrial dysfunction in psychiatric morbidity: current evidence and therapeutic prospects. Neuropsychiatr Dis Treat 2015;11:2441-7.

58. Penke B, Bogár F, Fülöp L. Protein folding and misfolding, endoplasmic reticulum stress in neurodegenerative diseases: in trace of novel drug targets. Curr Protein Pept Sci 2016;17:169-82.

59. Engin F, Hotamisligil GS. Restoring endoplasmic reticulum function by chemical chaperones: an emerging therapeutic approach for metabolic diseases. Diabetes Obes Metab 2010;12 Suppl 2:108-15.

60. Ricobaraza A, Cuadrado-Tejedor M, Marco S, Pérez-Otaño I, García-Osta A. Phenylbutyrate rescues dendritic spine loss associated with memory deficits in a mouse model of Alzheimer disease. Hippocampus 2012;22:1040-50.

61. Nunes AF, Amaral JD, Lo AC, Fonseca MB, Viana RJ, Callaerts-Vegh Z, et al. TUDCA, a bile acid, attenuates amyloid precursor protein processing and amyloid- $\beta$ deposition in
APP/PS1 mice. Mol Neurobiol 2012;45:440-54.

62. Wei H, Kim SJ, Zhang Z, Tsai PC, Wisniewski KE, Mukherjee AB. ER and oxidative stresses are common mediators of apoptosis in both neurodegenerative and non-neurodegenerative lysosomal storage disorders and are alleviated by chemical chaperones. Hum Mol Genet 2008;17:469-77.

63. Peng J, Liang G, Inan S, Wu Z, Joseph DJ, Meng Q, et al. Dantrolene ameliorates cognitive decline and neuropathology in Alzheimer triple transgenic mice. Neurosci Lett 2012;516:274-9.

64. Oseki KT, Monteforte PT, Pereira GJ, Hirata H, Ureshino RP, Bincoletto $\mathrm{C}$, et al. Apoptosis induced by $\mathrm{A} \beta 25-35$ peptide is $\mathrm{Ca}(2+)$ -IP3 signaling-dependent in murine astrocytes. Eur J Neurosci 2014;40:2471-8.

65. Gong T, Wang Q, Lin Z, Chen ML, Sun GZ. Endoplasmic reticulum (ER) stress inhibitor salubrinal protects against ceramide-induced SH-SY5Y cell death. Biochem Biophys Res Commun 2012; 427:461-5.

66. Patel S, Sharma D, Kalia K, Tiwari V. Crosstalk between endoplasmic reticulum stress and oxidative stress in schizophrenia: the dawn of new therapeutic approaches. Neurosci Biobehav Rev 2017;83:589-603.

67. Inokuchi Y, Nakajima Y, Shimazawa M, Kurita T, Kubo M, Saito A, et al. Effect of an inducer of BiP, a molecular chaperone, on endoplasmic reticulum (ER) stress-induced retinal cell death. Invest Ophthalmol Vis Sci 2009;50:334-44.

68. Shen YE, Wang Y, Yu GC, Liu C, Zhang ZY, Zhang LM. Effects of edaravone on amyloid- $\beta$ precursor protein processing in SY5YAPP695 cells. Neurotox Res 2013;24:139-47.

69. Fan J, Long H, Li Y, Liu Y, Zhou W, Li Q, et al. Edaravone protects against glutamate-induced PERK/EIF2 $\alpha / \mathrm{ATF} 4$ integrated stress response and activation of caspase-12. Brain Res 2013;1519:1-8.

70. Park GB, Kim YS, Lee HK, Song H, Cho DH, Lee WJ, et al. Endoplasmic reticulum stress-mediated apoptosis of EBV-transformed B cells by cross-linking of CD70 is dependent upon generation of reactive oxygen species and activation of p38 MAPK and JNK pathway. J Immunol 2010;185:7274-84. 